\title{
超高居里温度钙钛矿层状结构压电陶瓷研究进展
}

\author{
周志勇，陈 涛，董显林 \\ (中国科学院 上海硅酸盐研究所, 中国科学院无机功能材料与器件重点实验室, 上海 200050)
}

摘 要: 鿭铁矿层状压电陶瓷具有超高居里温度和高温度稳定性, 已成为目前高温压电陶瓷的研究热点。本文针对 钻钛矿层状压电陶瓷致密化烧结难以及压电性能低的难题, 主要从晶体结构、制备工艺、掺杂改性和复合固溶体等 方面总结了钻钛矿层状高温压电陶瓷的研究进展, 同时归纳和比较了不同制备工艺和掺杂改性的钻钛矿层状高温 压电陶瓷的烧结性和压电性能。简要分析了钻铁矿层状结构自发极化的来源, 并对未来研究这类材料的铁电相变机 理和提高压电性能作了展望。

关 键 词: 超高温压电陶瓷; 钻钛矿层状结构; 铁电体; 综述

中图分类号: TM282 文献标识码: A

\section{Research Progress of Perovskite Layer Structured Piezoelectric Ceramics with Super High Curie Temperature}

\author{
ZHOU Zhi-Yong, CHEN Tao, DONG Xian-Lin \\ (Key Laboratory of Inorganic Materials and Devices, Shanghai Institute of Ceramics, Chinese Academy of Sciences, Shanghai \\ 200050, China)
}

\begin{abstract}
Perovskite-layer structured (PLS) piezoelectric ceramics have the characteristics of ultra high Curie temperature and good thermal stability, thus PLS ceramics have become one of the hot topics in the field of high temperature piezoelectric ceramics. The present article reviews the research progress on PLS piezoelectric ceramics from the aspects of crystal structure, processing technologies, doping modifications, and forming solid solutions in order to overcome their disadvantages of poor sinterability and low piezoelectricity. Meanwhile, this review summarizes and compares the effects of processing technologies and doping modifications on the sinterability and piezoelectricity of PLS ceramics. Furthermore, the origin of the spontaneous polarization of PLS ferroelectircs is briefly described. The mechanism of ferroelectric phase transition and the approaches to improvement of piezoelectric properties for PLS piezoelectric ceramics are proposed for research work in the near future.
\end{abstract}

Key words: ultra high temperature piezoceramics; perovskite-layer structure; ferroelectrics; review

压电陶瓷是实现机械能与电能相互转换的一类 重要的信息功能材料, 可以制成各种压电传感器、 驱动器、换能器、谐振器和滤波器等电子元器件, 在 信息通讯、航空航天、汽车电子、医疗设备、石油 化工和军事等领域具有广泛的应用 ${ }^{[1-4]}$ 。
高温压电振动传感器是实现航空发动机、核反 应堆等关键装备振动参数实时测量的重要传感器, 其突出特点是服役条件苛刻, 工作温度高, 使用寿 命长 ${ }^{[5-7]}$ 。因此, 高温领域的应用对压电振动传感器 的核心敏感材料一高温压电陶瓷相应地提出了很高

收稿日期：2017-05-27; 收到修改稿日期：2017-06-30

基金项目: 中国科学院科技服务网络计划区域重点项目

Regional Key Program of Science and Technology Service Network Initiative Chinese Academy of Sciences

作者简介: 周志勇(1980-), 男, 副研究员. E-mail: zyzhou@mail.sic.ac.cn

通讯作者: 董显林, 研究员. E-mail: xldong@mail.sic.ac.cn 
的要求, 如高压电性能、高电阻率和高温度稳定性等。 压电陶瓷材料根据晶体结构分类，主要有钙钛 矿结构、铇青铜结构、铋层状结构和钙钛矿层状结 构(Perovskite-Layer Structured, PLS)压电陶瓷等 4 种。图 1 为 4 种不同结构压电陶瓷的压电系数 $d_{33}$ 与居里温度 $T_{\mathrm{c}}$ 之间的对应关系, 可以看出, 压电陶 瓷的 $d_{33}$ 与 $T_{\mathrm{C}}$ 既相互制约又相互依赖, $d_{33}$ 越高, $T_{\mathrm{c}}$ 则 越低, 反之亦然。

目前, 国际上高温压电振动传感器主要包括 $260^{\circ} \mathrm{C} 、 482^{\circ} \mathrm{C} 、 649^{\circ} \mathrm{C}$ 和 $760^{\circ} \mathrm{C}$ 等不同温度系列产品。 铋层状结构压电陶瓷是国际上 $482^{\circ} \mathrm{C}$ 压电传感器核 心敏感元件的通行解决方案。近年来, 针对铋层状 结构压电陶瓷的高温电阻率低和 $d_{33}$ 低等瓶颈问题, 四川大学、同济大学、中国科学院上海硅酸盐研究 所等单位开展了一系列研究工作并取得了重要进展 [8-11]。如四川大学通过优化组成设计和制备工艺等, 获 得了高压电性能 $\left(d_{33}: \sim 24 \mathrm{pC} / \mathrm{N}\right)$ 的 $\mathrm{CaBi}_{4} \mathrm{Ti}_{4} \mathrm{O}_{15}$ 基铋层 状结构压电陶瓷; 中国科学院上海硅酸盐研究所研 制的兼具高电阻率、高 $d_{33}$ 和高温度稳定性的 $\mathrm{CaBi}_{4} \mathrm{Ti}_{4} \mathrm{O}_{15}$ 基铋层状结构压电陶瓷, 已应用于我国 某系列型号航空发动机的振动测量, 并出口到美国 和韩国等国家。然而, 铋层状结构压电陶瓷的使用 温度上限在 $500^{\circ} \mathrm{C}$ 左右, 无法满足更高温度压电振 动传感器的使用需求。

PLS 压电陶瓷由于具有超高居里温度 $T_{\mathrm{c}}$ (如 $\mathrm{Sr}_{2} \mathrm{Nb}_{2} \mathrm{O}_{7}$ 的居里温度为 $1342^{\circ} \mathrm{C}^{[12]}, \mathrm{Pr}_{2} \mathrm{Ti}_{2} \mathrm{O}_{7}$ 的居里 温度高达 $1555^{\circ} \mathrm{C}^{[13]}$ )、高电阻率(比钙钛矿和铋层状 压电陶瓷以及 $\mathrm{LiNbO}_{3}$ 单晶高出 $2 \sim 4$ 个数量级 ${ }^{[7]}$ 以 及高温度稳定性 ${ }^{[14-15]}$ 等特点, 是 $600^{\circ} \mathrm{C}$ 以上高温

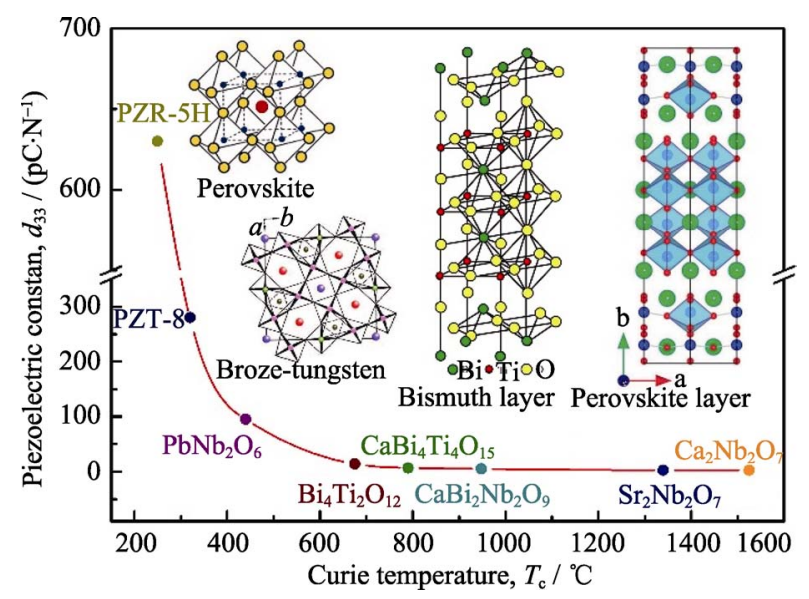

图 1 不同晶体结构压电陶瓷的压电系数 $d_{33}$ 与居里温度 $T_{\mathrm{c}}$ 之间的关系

Fig. 1 Relationship between $d_{33}$ and Curie temperature $T_{\mathrm{c}}$ of piezoceramics with different crystal structures

(Inset showing schematic diagrams of perovskite, tungsten bronze, bismuth layer and perovskite layer structures)
压电振动传感器用核心敏感元件的重要候选材料。 但是, PLS 压电陶瓷研制面临的关键技术问题如: (1) 普通烧结难以致密化(相对密度 $<92 \%)$; (2)矫顽场强 太高 $\left(E_{c}>10 \mathrm{kV} / \mathrm{mm}\right)$, 极化困难; (3)压电活性偏低 (压电系数 $d_{33}$ 在 $0 \sim 2 \mathrm{pC} / \mathrm{N}$ ), 这些因素极大地限制了 PLS 压电陶瓷在高温领域的实际应用。

因此，如何提高 PLS 压电陶瓷的烧结致密性和 压电性能一直是目前国际上研究的难点。近年来, 针对上述关键技术难题, 国内外研究机构所如英国 伦敦玛丽女王大学、中国科学院上海硅酸盐研究所、 中国工程物理研究院等相继在 PLS 压电陶瓷的制备 工艺、组成设计和性能优化等方面开展了大量研究 工作, 并取得了一系列重要进展, 为开发出新型高 温压电陶瓷奠定了基础。

本文将对超高居里温度 PLS 化合物的结构以及 PLS 压电陶瓷研究取得的主要进展进行简要介绍和 总结。

\section{1 钙钛矿层状结构(PLS)化合物}

\subsection{PLS 化合物概述}

对 PLS 化合物的研究可以追溯到半个多世纪以 前。1952 年, Cook 和 Jaffe ${ }^{[16]}$ 发现了一种在低温下具 有铁电性的 $\mathrm{A}_{2} \mathrm{~B}_{2} \mathrm{O}_{7}$ 型焦绿石结构。1955 年, Jona 等 ${ }^{[17]}$ 通过测定大量 $\mathrm{A}_{2} \mathrm{~B}_{2} \mathrm{O}_{7}$ 型化合物, 发现了焦绿 石结构 $\mathrm{Cd}_{2} \mathrm{Nb}_{2} \mathrm{O}_{7}$ 。 1958 年, Rowland 等 ${ }^{[18]}$ 发现了 $\mathrm{Ca}_{2} \mathrm{Nb}_{2} \mathrm{O}_{7}$ 存在一个不同于焦绿石结构的解理面和 氧八面体框架, 但没有铁电性。由于 $\mathrm{Cd}^{2+}$ 和 $\mathrm{Ca}^{2+}$ 具 有相似的离子半径, 两种化合物有可能具有相似的 结构和电学性质。1965 年, Ballman 等 ${ }^{[19]}$ 从实验中发 现, $\mathrm{Ca}_{2} \mathrm{Nb}_{2} \mathrm{O}_{7}$ 在液氮温度以上不具有铁电性。1970 年, Brandon 等 ${ }^{[20]}$ 通过 $X$ 射线衍射方法发现, $\mathrm{A}_{2} \mathrm{~B}_{2} \mathrm{O}_{7}$ 型化合物由畸变的钙钛矿构成层状结构, 它们被归 为两种不同的结构:第一种类似于 $\mathrm{Cd}_{2} \mathrm{Nb}_{2} \mathrm{O}_{7}$ 的焦绿 石结构, 钙钛矿层平行于(110)方向; 第二种结构的 钙钛矿层平行于 $(100)$ 方向, 如 $\mathrm{Ca}_{2} \mathrm{Nb}_{2} \mathrm{O}_{7}$ 。在随后的 研究中, 研究者相继发现了另外 5 种与 $\mathrm{Ca}_{2} \mathrm{Nb}_{2} \mathrm{O}_{7}$ 结

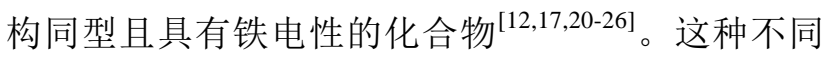
于焦绿石的结构被定义为钙钛矿层状结构(PLS), 表 1 总结了部分 $\mathrm{A}_{2} \mathrm{~B}_{2} \mathrm{O}_{7}$ 型化合物结构及其铁电性。

到目前为止, 已发现的具有铁电性的 $\mathrm{A}_{2} \mathrm{~B}_{2} \mathrm{O}_{7}$ 型 PLS 化合物共有 7 种: $\mathrm{Ca}_{2} \mathrm{Nb}_{2} \mathrm{O}_{7} 、 \mathrm{Sr}_{2} \mathrm{Nb}_{2} \mathrm{O}_{7}$ 、 $\mathrm{Sr}_{2} \mathrm{Ta}_{2} \mathrm{O}_{7} 、 \mathrm{La}_{2} \mathrm{Ti}_{2} \mathrm{O}_{7} 、 \mathrm{Nd}_{2} \mathrm{Ti}_{2} \mathrm{O}_{7} 、 \mathrm{Pr}_{2} \mathrm{Ti}_{2} \mathrm{O}_{7}$ 和 $\mathrm{Ce}_{2} \mathrm{Ti}_{2} \mathrm{O}_{7}$, 而具有铁电性的 $\mathrm{A}_{2} \mathrm{~B}_{2} \mathrm{O}_{7}$ 型焦绿石结构化合物只有 $\mathrm{Cd}_{2} \mathrm{Nb}_{2} \mathrm{O}_{7}$ 。值得注意的是, 1980 年 Sych 等 ${ }^{[24]}$ 报道了 $\mathrm{Ce}_{2} \mathrm{Ti}_{2} \mathrm{O}_{7}$ 陶瓷具有焦绿石结构, 但没有铁电性; 然 
表 $1 \quad \mathbf{A}_{2} \mathbf{B}_{2} \mathbf{O}_{7}$ 型化合物和结构

Table $1 \quad A_{2} B_{2} O_{7}$-type compounds and their structures

\begin{tabular}{ccccc}
\hline PY* & Formula & Structure & Ferroelectric & Ref. \\
\hline 1955 & $\mathrm{Cd}_{2} \mathrm{Nb}_{2} \mathrm{O}_{7}$ & Pyrochlore & Yes & {$[17]$} \\
1955 & $\mathrm{Ca}_{2} \mathrm{Ta}_{2} \mathrm{O}_{7}$ & Pyrochlore & $\mathrm{No}$ & {$[17]$} \\
1955 & $\mathrm{Cd}_{2} \mathrm{Ta}_{2} \mathrm{O}_{7}$ & Pyrochlore & No & {$[17]$} \\
1955 & $\mathrm{~Pb}_{2} \mathrm{Ta}_{2} \mathrm{O}_{7}$ & Pyrochlore & No & {$[17]$} \\
1970 & $\mathrm{Ca}_{2} \mathrm{Nb}_{2} \mathrm{O}_{7}$ & PLS & Yes & {$[19]$} \\
1974 & $\mathrm{La}_{2} \mathrm{Ti}_{2} \mathrm{O}_{7}$ & PLS & Yes & {$[21]$} \\
1974 & $\mathrm{Nd}_{2} \mathrm{Ti}_{2} \mathrm{O}_{7}$ & PLS & Yes & {$[22]$} \\
1975 & $\mathrm{Sr}_{2} \mathrm{Nb}_{2} \mathrm{O}_{7}$ & PLS & Yes & {$[23]$} \\
1975 & $\mathrm{Sr}_{2} \mathrm{Ta}_{2} \mathrm{O}_{7}$ & PLS & Yes & {$[12]$} \\
1980 & $\mathrm{Pr}_{2} \mathrm{Ti}_{2} \mathrm{O}_{7}$ & PLS & Yes & {$[24]$} \\
1980 & $\mathrm{Ce}_{2} \mathrm{Ti}_{2} \mathrm{O}_{7}$ & Pyrochlore & No & {$[24]$} \\
1987 & $\mathrm{Sm}_{2} \mathrm{Ti}_{2} \mathrm{O}_{7}$ & Pyrochlore & No & {$[25]$} \\
2015 & $\mathrm{Ce}_{2} \mathrm{Ti}_{2} \mathrm{O}_{7}$ & PLS & Yes & {$[26]$} \\
\hline
\end{tabular}

*PY: Publication Year

而, 2015 年 $\mathrm{Gao}$ 等 ${ }^{[26]}$ 利用 $4 \mathrm{GPa}$ 高压力成功制备了 单相 $\mathrm{Ce}_{2} \mathrm{Ti}_{2} \mathrm{O}_{7}$ 陶瓷, 其压电系数 $d_{33}$ 为 $0.4 \mathrm{pC} / \mathrm{N}$, 并 获得了电滞回线的电流峰, 表明 $\mathrm{Ce}_{2} \mathrm{Ti}_{2} \mathrm{O}_{7}$ 具有铁电 性。不过这一结果还要更多的结构分析和实验证据。

\subsection{PLS 化合物晶体结构}

PLS 化合物的结构通式为 $\mathrm{A}_{n} \mathrm{~B}_{n} \mathrm{O}_{3 n+2}$, 它是由共 享顶角氧原子的氧八面体 $\mathrm{BO}_{6}$ 和 12 配位的阳离子 $\mathrm{A}$ 构成的畸变钙钛矿结构层状堆叠而成。 $n$ 表示钙钛 矿层中氧八面体的个数, 当 $n=4$ 时, 化简得到 $\mathrm{A}_{2} \mathrm{~B}_{2} \mathrm{O}_{7}$ 型结构; 当 $n=\infty$ 时, 化简得到 $\mathrm{ABO}_{3}$ 型钙钛 矿结构, 所以可以将钙钠矿层状结构理解为钙钛矿 结构沿某个特定方向切割形成的层状结构 ${ }^{[23]}$ 。表 2 总结了 $\mathrm{A}_{2} \mathrm{~B}_{2} \mathrm{O}_{7}$ 型 PLS 化合物晶体结构、空间点群 和晶胞参数等 ${ }^{[21-22,27-30]}$ 。

PLS 化合物主要有三种晶体结构 ${ }^{[20,23,30]}$ : 解理 面为 (010)的正交结构, 解理面为(100)的单斜结构 和解理面为(001)的单斜结构。以(010)正交结构
$\mathrm{Sr}_{2} \mathrm{Nb}_{2} \mathrm{O}_{7}$ 为例, 其低温铁电相为正交结构, 空间群 为 $\mathrm{Cmc}_{1}$, 图 2(a)和(b)分别为沿 $a$ 轴和 $c$ 轴方向投 影的晶体结构示意图, $a$ 轴为氧八面体链的方向, 钙 钛矿层状畸变可以理解成氧八面体 $\mathrm{NbO}_{6}$ 有一个小 角度旋转, $b$ 轴垂直于钙钛矿层的方向, $c$ 轴为极化 方向, 层与层之间多出额外的 Sr-O 层, 并沿 $a$ 方向 平移了 $a / 2$ 的距离。

$\mathrm{Ca}_{2} \mathrm{Nb}_{2} \mathrm{O}_{7} 、 \mathrm{La}_{2} \mathrm{Ti}_{2} \mathrm{O}_{7}$ 和 $\mathrm{Nd}_{2} \mathrm{Ti}_{2} \mathrm{O}_{7}$ 空间点群相同 ${ }^{[23]}$, 其结构与 $\mathrm{Sr}_{2} \mathrm{Nb}_{2} \mathrm{O}_{7}$ 相似, 不同的是这种结构为单斜 晶系, 其解理方向为(100)方向。 $\operatorname{Pr}_{2} \mathrm{Ti}_{2} \mathrm{O}_{7}$ 属于单斜 晶系，但其解理方向为(001)方向 ${ }^{[30]}$ 。

\section{PLS 化合物第一性原理计算}

对于 $\mathrm{ABO}_{3}$ 钙铁矿结构铁电体, 一般为 $\mathrm{B}$ 位离 子驱动(如 $\mathrm{BaTiO}_{3}$ ), $\mathrm{B}$ 位由含有空位的 $\mathrm{d}$ 轨道的过渡 金属元素构成，因为 $\mathrm{d}^{0}$ 轨道容易与氧原子的 $2 \mathrm{p}$ 轨 道杂化, 使 $\mathrm{B}$ 位原子发生偏移振动, 从而产生自发 极化 ${ }^{[31-32]}$ 。另外, 还有少量的 $\mathrm{A}$ 位离子驱动铁电体, 如在 $\mathrm{BiFeO}_{3}$ 中, $\mathrm{Bi}$ 含有 $6 \mathrm{~s}$ 孤对电子, 容易与其他轨 道杂化, 使 $\mathrm{A}$ 位原子偏离, 形成自发极化 ${ }^{[33]}$ 。从晶 胞结构来看, 虽然钙钠矿结构和钙钛矿层状结构都 只包含了氧八面体，但是后者的自发极化、结构相 变等内在物理机制要比前者复杂得多, 到目前为止 相关的理论计算还较少，也没有得到实验验证。

Jorege 等 ${ }^{[34]}$ 通过第一性原理计算了 $\mathrm{La}_{2} \mathrm{Ti}_{2} \mathrm{O}_{7}$ 铁 电顺电相变过程, 计算结果表明 $\mathrm{La}_{2} \mathrm{Ti}_{2} \mathrm{O}_{7}$ 是氧八面 体驱动型铁电体, 原子位移主要由氧原子贡献, $\mathrm{B}$ 位 $\mathrm{Ti}^{4+}$ 对自发极化几乎没有贡献。如图 3 所示, 箭 头表示氧原子位移产生的电偶极矩, 图 3(a)为 PLS 结构, 总电偶极矩为 $\boldsymbol{p}^{\text {layer }}=2 \boldsymbol{p}_{1}+2 \boldsymbol{p}_{3}+\boldsymbol{p}_{5}$, 因此氧原子 产生的净电偶极矩不为零, 从而表现出宏观极化 率。而对于钙钛矿结构(图 3(b)), 连续的氧八面体造 成氧原子产生的电偶极矩相互抵消, 对极化率的贡 献为零。

表 $2 \quad \mathbf{A}_{2} \mathbf{B}_{2} \mathbf{O}_{7}$ 型 PLS 化合物的晶体特征

Table 2 Crystallographic properties of $\mathrm{A}_{2} \mathrm{~B}_{2} \mathrm{O}_{7}$-type PLS compounds

\begin{tabular}{|c|c|c|c|c|c|c|c|c|}
\hline \multirow{2}{*}{ Compound } & \multirow{2}{*}{ Crystal system } & \multirow{2}{*}{ Space group } & \multicolumn{4}{|c|}{ Lattice constants } & \multirow{2}{*}{ Cleavage plane } & \multirow{2}{*}{ Ref. } \\
\hline & & & $a / \mathrm{nm}$ & $b / \mathrm{nm}$ & $c / \mathrm{nm}$ & $\beta$ & & \\
\hline $\mathrm{Sr}_{2} \mathrm{Nb}_{2} \mathrm{O}_{7}$ & Orthorhombic & $\mathrm{Cmc} 2_{1}$ & 0.3933 & 2.6726 & 0.5683 & - & $(010)$ & [27] \\
\hline $\mathrm{Sr}_{2} \mathrm{Ta}_{2} \mathrm{O}_{7}$ & Orthorhombic & $\mathrm{Cmc}_{1}$ & 0.3950 & 2.7270 & 0.5700 & - & $(010)$ & [28] \\
\hline $\mathrm{Ca}_{2} \mathrm{Nb}_{2} \mathrm{O}_{7}$ & Monoclinic & $\mathrm{P} 2_{1}$ & 1.3400 & 0.5510 & 0.7720 & $98^{\circ} 17^{\prime}$ & (100) & [29] \\
\hline $\mathrm{La}_{2} \mathrm{Ti}_{2} \mathrm{O}_{7}$ & Monoclinic & $\mathrm{P} 2_{1}$ & 1.3019 & 0.5547 & 0.7811 & $98^{\circ} 43^{\prime}$ & (100) & [21] \\
\hline $\mathrm{Nd}_{2} \mathrm{Ti}_{2} \mathrm{O}_{7}$ & Monoclinic & $\mathrm{P} 2_{1}$ & 1.3020 & 0.5480 & 0.7680 & $98^{\circ} 28^{\prime}$ & $(100)$ & [22] \\
\hline $\mathrm{Pr}_{2} \mathrm{Ti}_{2} \mathrm{O}_{7}$ & Monoclinic & $\mathrm{P} 2_{1}$ & 0.7715 & 0.5488 & 1.3004 & $98^{\circ} 33^{\prime}$ & $(001)$ & [30] \\
\hline
\end{tabular}




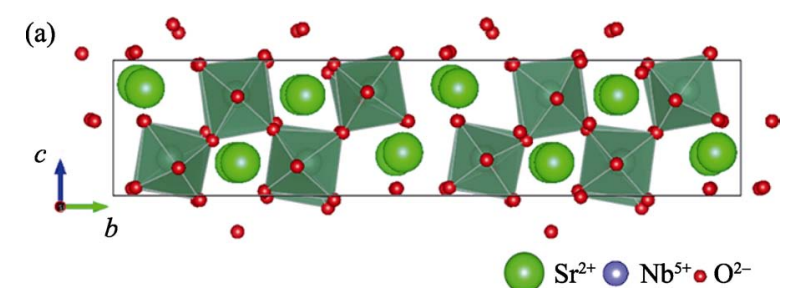

(b)

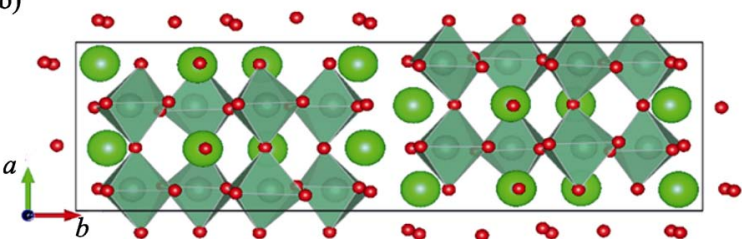

图 2 (a)沿 $a$ 轴和(b)沿 $c$ 轴方向 $\mathrm{Sr}_{2} \mathrm{Nb}_{2} \mathrm{O}_{7}$ 晶体结构示意图 Fig. 2 Crystal structure of $\mathrm{Sr}_{2} \mathrm{Nb}_{2} \mathrm{O}_{7}$ viewed along $a$ (a) and along $c(b)$

Atoms are labelled in accordance with the nomenclature of Ref [23]

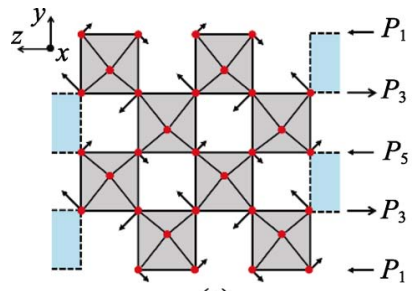

(a)

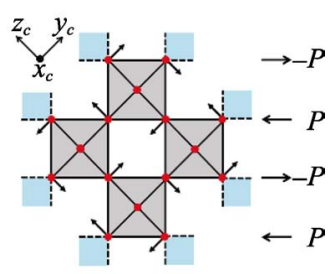

(b)
图 3 (a) $\mathrm{Cmcm}$ 相 $\mathrm{La}_{2} \mathrm{Ti}_{2} \mathrm{O}_{7}$ 中最不稳态模式下最大原子位移 示意图和(b)理想钙钛矿结构 $\mathrm{BaTiO}_{3}$ 中典型反铁电畸变模式 示意图。箭头表示不同 $y$ 面上与氧位移相关的电偶极矩 ${ }^{[34]}$

Fig. 3 (a) Sketch of the largest atomic displacements associated with the strongest instability mode obtained for the $\mathrm{Cmcm}$ phase of $\mathrm{La}_{2} \mathrm{Ti}_{2} \mathrm{O}_{7}$; (b) Sketch of a typical anti-ferrodistortive mode occurring in an ideal perovskite structure of $\mathrm{BaTiO}_{3}$. The arrows on the side represent the electric dipoles associated to the displacement of oxygens in different $y$-planes ${ }^{[34]}$

Bruyer 等 ${ }^{[35]}$ 用第一性原理研究了 $\mathrm{La}_{2} \mathrm{Ti}_{2} \mathrm{O}_{7}$ 和 $\mathrm{Nd}_{2} \mathrm{Ti}_{2} \mathrm{O}_{7}$ 化合物的电子结构和铁电性, 理论计算两 者的自发极化率分别为 $7.72 \mu \mathrm{C} / \mathrm{cm}^{2}$ 和 $7.42 \mu \mathrm{C} / \mathrm{cm}^{2}$ 。 图 4 所示为 $\mathrm{La}_{2} \mathrm{Ti}_{2} \mathrm{O}_{7}$ 化合物的电子态密度分布图, 可以看到, $\mathrm{Ti}$ 和 $\mathrm{O}$ 之间不是单一的离子键, 还存在 较强的共价键，铁电相在 Ti4d 和 O2p 轨道之间的杂 化增强, 导致电子态密度分布各向异性。但没有计 算铁电相变对 $\mathrm{TiO}_{6}$ 八面体的电子态密度分布影响。 因此, 对 PLS 化合物自发极化的研究可以通过计 算铁电顺电相变的氧八面体电子态密度、氧原子位 移和旋转模式以及高分辨 TEM 实际观测来证实。

\section{PLS 压电陶瓷制备工艺与性能研究}

PLS 压电陶瓷的晶粒是典型的片状形貌结构, 片状晶粒随机生长易导致晶粒之间形成孔隙, 因此 通过普通固相反应法难以实现致密化烧结(致密度

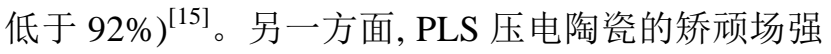
太高, 其晶体结构特点决定了其极化方向单一, 造

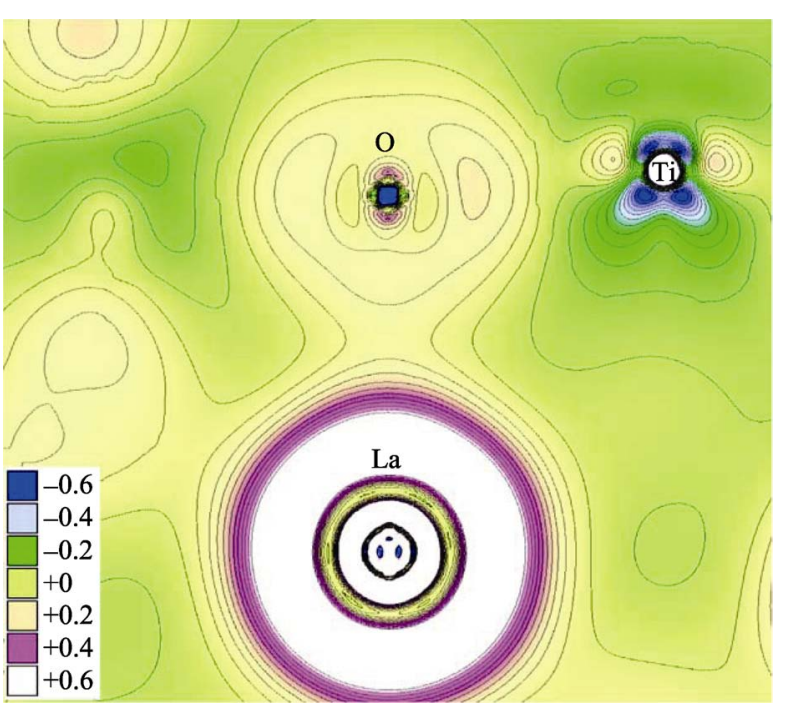

图 4 计算的单斜 $\mathrm{P}_{1} \mathrm{La}_{2} \mathrm{Ti}_{2} \mathrm{O}_{7}$ 化合物价电子电荷密度。等 高线相差为 $0.05 e \AA^{-3 \text { [35] }}$

Fig. 4 Calculated valence-electron charge density for the monoclinic $\mathrm{P}_{2} \mathrm{La}_{2} \mathrm{Ti}_{2} \mathrm{O}_{7}$. Contour lines differ by $0.05 e \AA^{-3 \text { [35] }}$ ( $1 \AA=0.1 \mathrm{~nm})$

成压电活性很低 ${ }^{[14]}$ 。近几年开展的研究工作主要围 绕制备工艺、组成设计和性能优化等方面。

\section{1 制备工艺}

PLS 压电陶瓷粉体的合成有固相反应合成、熔 盐合成 ${ }^{[36]}$ 和溶胶-凝胶合成法 ${ }^{[37]}$ 。PLS 压电陶瓷粉体 的烧结主要包括普通固相反应烧结(Solid State Reaction, SSR)、放电等离子体烧结(Spark Plasma Sintering, SPS)和热锻烧结(Hot Forging, HF)等。

PLS 压电陶瓷的片状晶粒具有各向异性的特点, 因此, 可以通过织构工艺减小晶粒之间的孔隙, 提 高致密度和取向度，进而提高压电性能。2009 年, Yan 等 ${ }^{[14]}$ 首次采用 SPS 法制备了具有高度晶粒取向 的 $\mathrm{Nd}_{2} \mathrm{Ti}_{2} \mathrm{O}_{7}$ 和 $\mathrm{La}_{2} \mathrm{Ti}_{2} \mathrm{O}_{7}$ 压电陶瓷, 其致密度均达到 $98 \%$ 以上, 并测得其居里温度分别为 $1482^{\circ} \mathrm{C}$ 和 $1461^{\circ} \mathrm{C}, d_{33}$ 为 $2.6 \mathrm{pC} / \mathrm{N}$ 。Fuierer 等 ${ }^{[38]}$ 采用 $\mathrm{HF}$ 方法 制备了 $\mathrm{Sr}_{2} \mathrm{NbTaO}_{7}$ 陶瓷, 致密度提高至 $95 \%, d_{33}$ 达 $1.6 \mathrm{pC} / \mathrm{N}$ 。Gao 等 ${ }^{[39]}$ 利用 $12 \mathrm{~T}$ 磁场辅助注浆成型、 结合模板晶粒生长法, 最后通过固相反应烧结制备了 晶粒取向度为 0.76 、致密度达 $95 \%$ 的 $\mathrm{La}_{2} \mathrm{Ti}_{2} \mathrm{O}_{7}$ 陶瓷。

促进致密化烧结的另一个有效方法就是添加适 量烧结助剂。Seraji 和 Iqbal 等 ${ }^{[40-41]}$ 在 $\mathrm{Sr}_{2} \mathrm{Nb}_{2} \mathrm{O}_{7}$ 中分 别添加适量 $\mathrm{V}_{2} \mathrm{O}_{5}$ 和 $\mathrm{ZnO}$, 通过固相烧结法将其致密 度提高至 $96 \%$, 但没有报道压电系数。最近董显林 课题组 ${ }^{[42]}$ 在 $\mathrm{Sr}_{2} \mathrm{Nb}_{2} \mathrm{O}_{7}$ 中加入 $0.5 \mathrm{wt} \% \mathrm{CuO}$, 将陶瓷烧 结温度从 $1420^{\circ} \mathrm{C}$ 大幅降低至 $1180^{\circ} \mathrm{C}$, 致密度显著提 高到 98\%, 并通过施加高极化电场( $18 \mathrm{kV} / \mathrm{mm}), d_{33}$ 达到 $1.1 \mathrm{pC} / \mathrm{N}$, 且 $T_{\mathrm{c}}$ 保持不变 $\left(1342^{\circ} \mathrm{C}\right)$ 。这些结果 表明, 添加合适的烧结助剂, 不仅可以有效改善 
PLS 压电陶瓷的烧结行为, 提高致密度, 而且可以 优化其电学性能。最近, Gao 等 ${ }^{[26]}$ 发展了一种新型制 备方法, 在 $4 \mathrm{GPa}$ 高压力下成功制备了单相 $\mathrm{Ce}_{2} \mathrm{Ti}_{2} \mathrm{O}_{7}$ 陶瓷，其压电系数 $d_{33}$ 为 $0.4 \mathrm{pC} / \mathrm{N}$ 。

图 5 对比了几种典型工艺制备的不同 PLS 压电 陶瓷的形貌。从现有的研究结果来看, PLS 压电陶瓷 的致密化烧结难题已可以通过不同工艺手段得到不 同程度的解决，致密度可达 $98 \%$ 以上，这为施加更 高极化电场、获得更高压电性能提供了前提条件。

\section{$3.2 \mathrm{~A} / \mathrm{B}$ 位掺杂取代}

在压电陶瓷材料研究中, 合适的离子掺杂取代 是提高压电性能的主要途径。从现有研究结果来看, 有压电性能的 PLS 压电陶瓷主要是采用 SPS 制备的, 仅通过离子掺杂取代结合普通烧结工艺还难以获得 高压电性能。如 2009 年 Yan 等 ${ }^{[14]}$ 通过 SPS 工艺制 备了高致密( $>98 \%)$ 、高取向度( 0.82$)$ 的 $\mathrm{Nd}_{2} \mathrm{Ti}_{2} \mathrm{O}_{7}$ 和 $\mathrm{La}_{2} \mathrm{Ti}_{2} \mathrm{O}_{7}$ 压电陶瓷， $d_{33}$ 高达 $2.6 \mathrm{pC} / \mathrm{N}$ 。而 $\mathrm{Fu}$ 等 ${ }^{[43]}$ 通过 $\mathrm{La}$ 掺杂制备了致密 $\mathrm{Sr}_{2} \mathrm{Nb}_{2} \mathrm{O}_{7}$ 压电陶瓷, 但没有报 道压电系数。2017 年 $\mathrm{Li}$ 等 ${ }^{[44]}$ 用 $\mathrm{Ba}^{2+}$ 取代 $\mathrm{Ca}_{2} \mathrm{Nb}_{2} \mathrm{O}_{7}$ 中 A 位, 通过 XRD 和拉曼光谱研究发现固溶极限为 0.3 , 居里温度明显下降, 但仍然没有报道其压电系数。

因此, 目前的研究工作主要集中在通过离子掺 杂与 SPS 工艺相结合的方法, 获得兼具有高致密度 和一定压电性能的 PLS 压电陶瓷。Gao 等 ${ }^{[45]}$ 利用 SPS 工艺制备了 $\mathrm{Sr}_{2-x} \mathrm{Ba}_{x} \mathrm{Nb}_{2} \mathrm{O}_{7}$ 压电陶瓷, $\mathrm{Ba}^{2+}$ 掺杂 可将 $d_{33}$ 提高至 $3.6 \mathrm{pC} / \mathrm{N}$ ，同时利用非原位退火实验 表明热退极化温度高于 $1150^{\circ} \mathrm{C}$, 如图 6 所示。他们 进一步通过 SPS 制备了织构化的 $\mathrm{Ce}^{3+}$ 掺杂 $\mathrm{La}_{2} \mathrm{Ti}_{2} \mathrm{O}_{7}$ 陶瓷，成功将 $d_{33}$ 提高到 $3.9 \mathrm{pC} / \mathrm{N}$, 这是目前报道

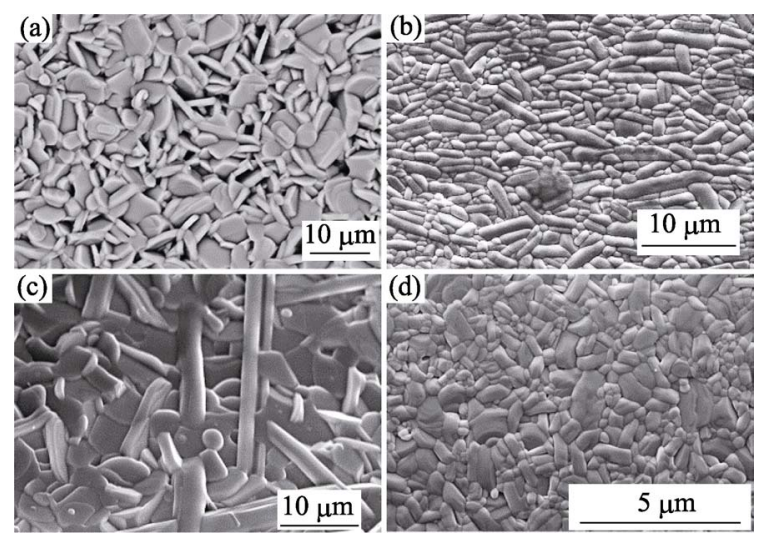

图 5 不同工艺在制备的 PLS 高温压电陶瓷 SEM 照片

Fig. 5 SEM images of PLS high temperature piezoceramics fabricated with different techniques

(a) $\mathrm{Sr}_{2} \mathrm{Nb}_{2} \mathrm{O}_{7}$ by solid state reaction method ${ }^{[42]}$; (b) $\mathrm{Sr}_{2} \mathrm{Nb}_{2} \mathrm{O}_{7}$ by spark plasma sintering ${ }^{[15]}$; (c) $0.3 \mathrm{wt} \% \mathrm{ZnO}-\mathrm{Sr}_{2} \mathrm{Nb}_{2} \mathrm{O}_{7}$ by solid state reaction method ${ }^{[41]}$; (d) $\mathrm{Ce}_{2} \mathrm{Ti}_{2} \mathrm{O}_{7}$ at $4 \mathrm{GPa}, 1100{ }^{\circ} \mathrm{C}^{[26]}$

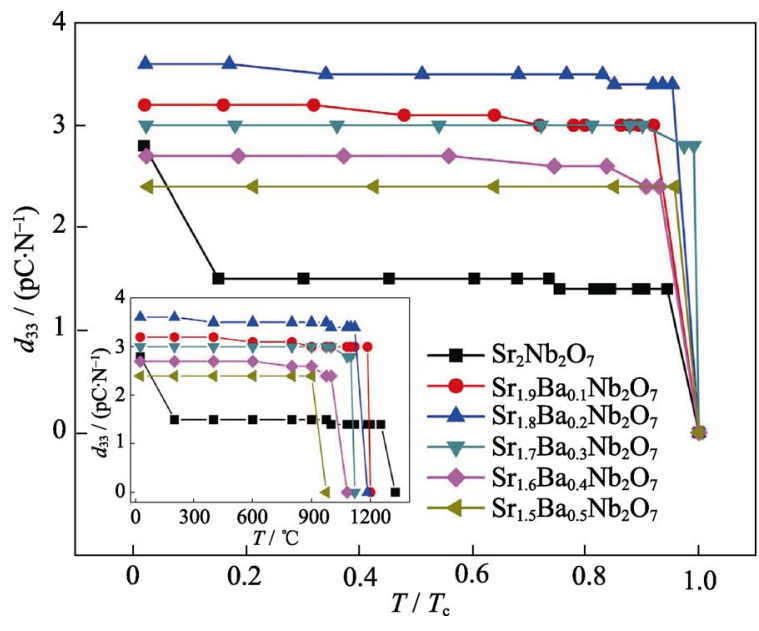

图 6 不同温度下热退极化对 $\mathrm{Sr}_{2-x} \mathrm{Ba}_{x} \mathrm{Nb}_{2} \mathrm{O}_{7}$ 压电陶瓷压电 性能的影响 ${ }^{[45]}$

Fig. 6 Effect of thermal depoling on piezoelectric properties of $\mathrm{Sr}_{2-\chi} \mathrm{Ba}_{x} \mathrm{Nb}_{2} \mathrm{O}_{7}$ piezoceramics ${ }^{[45]}$

的 PLS 压电陶瓷 $d_{33}$ 的最高值 ${ }^{[46]}$ 。随后, Ning 等 ${ }^{[47]}$ 用 SPS 方法制备了 $\mathrm{Ce}^{3+}$ 和 $\mathrm{W}^{6+}$ 掺杂的 $\mathrm{Sr}_{2} \mathrm{Nb}_{2} \mathrm{O}_{7}$ 压电 陶瓷, 结果表明 $\mathrm{A}$ 位 $\mathrm{Ce}^{3+}$ 取代测得压电系数为 1.5 $\mathrm{pC} / \mathrm{N}, \mathrm{B}$ 位 $\mathrm{W}^{6+}$ 取代并未测得压电系数。Chen 等 ${ }^{[88]}$ 用 SPS 方法制备了 $\mathrm{Sm}^{3+} / \mathrm{Eu}^{3+}$ 取代的 $\mathrm{La}_{2} \mathrm{Ti}_{2} \mathrm{O}_{7}$ 压电 陶瓷，发现 $\mathrm{Sm}^{3+}$ 和 $\mathrm{Eu}^{3+}$ 的固溶极限分别为 0.8 和 0.5 , 测得最佳组分为 $\left(\mathrm{Sm}_{0.1} \mathrm{La}_{0.9}\right) \mathrm{Ti}_{2} \mathrm{O}_{7}$ 的 $d_{33}$ 最高为 $2.8 \mathrm{pC} / \mathrm{N}$, 并获得了电滞回线的电流峰。 $\mathrm{Li}$ 等 ${ }^{[49]}$ 通过 SPS 方法 制备了 $\left(\mathrm{Bi}_{x} \mathrm{La}_{1-x}\right) \mathrm{Ti}_{2} \mathrm{O}_{7}$, 测得 $d_{33}$ 最高为 $3.6 \mathrm{pC} / \mathrm{N}$, 压 电性的提高是由于 $\mathrm{Bi}^{3+}$ 存在 $6 \mathrm{~s}$ 孤对电子, 对极化率 有一定的贡献。

\section{3 复合固溶体}

压电陶瓷中通常通过添加第二组元的方法提高 材料的烧结性、压电以及介电性能。一方面, 多种 化合物固溶的过程使体系自由能降低, 有利于烧结; 另一方面, 随着添加第二组元含量的变化, 相结构 也随之变化, 从而形成准同型相界(MPB)或多形态 相变 $(\mathrm{PPT})$ 。Titov 等 ${ }^{[50]}$ 将钙钛矿 $\mathrm{NaNbO}_{3}$ 引入 $\mathrm{Ca}_{2} \mathrm{Nb}_{2} \mathrm{O}_{7}$ 和 $\mathrm{Sr}_{2} \mathrm{Nb}_{2} \mathrm{O}_{7}$ 中, 通过分型晶体结构的演变 设计一种新型的铌酸盐铁电体, 但没有报道材料体 系的压电介电性能。2016 年, 董显林课题组 ${ }^{[51]}$ 将钙钛 矿 $\mathrm{Na}_{0.5} \mathrm{Bi}_{0.5} \mathrm{TiO}_{3}$ 引入到 $\mathrm{Sr}_{2} \mathrm{Nb}_{2} \mathrm{O}_{7}$ 中, 采用普通固相 反应烧结法制备了 $(1-x) \mathrm{Sr}_{2} \mathrm{Nb}_{2} \mathrm{O}_{7}-x\left(\mathrm{Na}_{0.5} \mathrm{Bi}_{0.5}\right) \mathrm{TiO}_{3}$ $(x=0 \sim 0.05)$ 压电陶瓷, $\mathrm{Na}_{0.5} \mathrm{Bi}_{0.5} \mathrm{TiO}_{3}$ 的引入较好地改 善了 $\mathrm{Sr}_{2} \mathrm{Nb}_{2} \mathrm{O}_{7}$ 陶瓷的烧结性能, 使致密度从 $92 \%$ 提 高到 96.8\%, 并首次测得了非织构 $\mathrm{Sr}_{2} \mathrm{Nb}_{2} \mathrm{O}_{7}$ 基陶瓷 的压电性能 $\left(d_{33}: \sim 1.0 \mathrm{pC} / \mathrm{N}\right)$, 且具有很好的温度稳 定性, 退极化温度在 $1200^{\circ} \mathrm{C}$ 以上(如图 7 所示)。 


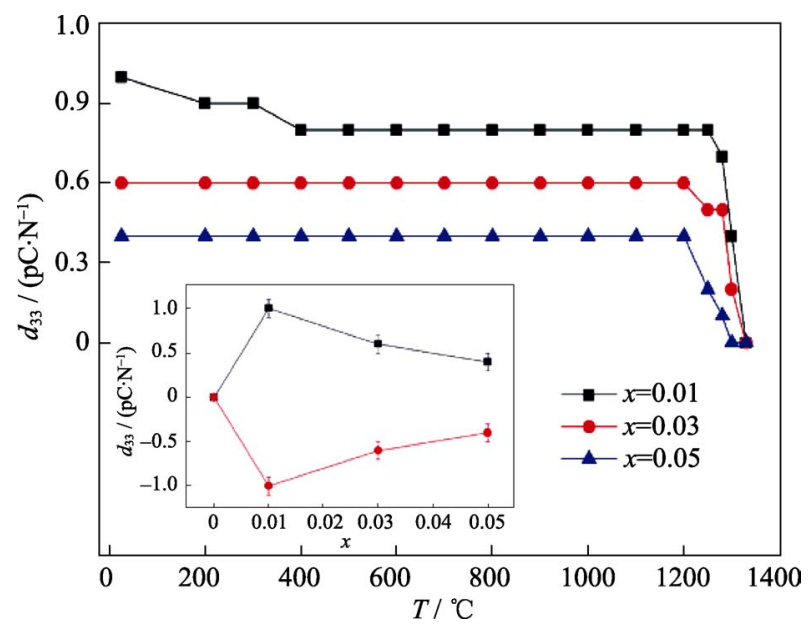

图 7 不同温度下热退极化对 $(1-x) \mathrm{Sr}_{2} \mathrm{Nb}_{2} \mathrm{O}_{7}-x\left(\mathrm{Na}_{0.5} \mathrm{Bi}_{0.5}\right) \mathrm{TiO}_{3}$ 压 电陶瓷 $d_{33}$ 的影响 ${ }^{[51]}$

Fig. 7 Temperature dependence of $d_{33}$ values of

$(1-x) \mathrm{Sr}_{2} \mathrm{Nb}_{2} \mathrm{O}_{7}-x\left(\mathrm{Na}_{0.5} \mathrm{Bi}_{0.5}\right) \mathrm{TiO}_{3}$ piezoceramics after thermal annealing $^{[51]}$

表 3 总结了近年来不同方法制备的 PLS 压电陶 瓷的主要性能, 可以看出, 目前主要是采用 SPS 烧 结与离子掺杂相结合的方法来提高 PLS 压电陶瓷的 烧结性能和压电性能, 但最近普通固相烧结法已取 得较大的进展, 尽管与 SPS 制备的陶瓷相比, 还有 一定的差距。

\section{4 结语与展望}

超高居里温度 PLS 压电陶瓷的致密化烧结难题 已可以通过不同工艺手段得到相当程度的解决,
致密度可高达 98\%以上，这为施加更高极化电场、 获得更好压电性能提供了前提条件。

针对压电系数低的难题, 研究工作主要集中在 通过离子掺杂与 SPS 工艺相结合的方法, 获得兼具 高致密度和一定压电性能的 PLS 压电陶瓷。最近, 采用普通固相反应法烧结, 通过添加 $\mathrm{CuO}$ 或复合固 溶体使 PLS 压电陶瓷的 $d_{33}$ 达到 $2 \mathrm{pC} / \mathrm{N}$ 左右, 取得 了突破。虽然通过工艺改进、掺杂取代和添加第二 组元等方法在一定程度上提高了 PLS 压电陶瓷的压 电性能, 但是, 其压电系数仍然偏小, 还难以满足 高温压电振动传感器的使用需求。

一方面, 从材料组成设计角度, 通过引入压电 性能较好的铋层状压电材料为第二相, 构建钙钛矿 层状-铋层状结构复合材料, 以期获得高居里温度、 高压电性能的压电陶瓷材料; 另一方面, 有必要从 铁电体自发极化的物理本质出发, 探究 PLS 化合物 的铁电顺电相变机理以及晶体结构对自发极化的影 响。如 $\mathrm{Gao}$ 等 ${ }^{[52]}$ 计算了 $\mathrm{Nd}_{2} \mathrm{Ti}_{2} \mathrm{O}_{7}$ 各原子对自发极化 的贡献, 发现自发极化主要来自于氧原子的贡献。 然而，至今还没有通过实验观测到 PLS 化合物中氧 原子位移、铁电畴的运动以及证明自发极化来自于 氧八面体畸变。并且, 大量的实验证明 $\mathrm{A}$ 位取代对 于压电性能的影响比 B 位取代更大。

因此, 将来对于 PLS 压电陶瓷的研究可能集中 在: 第一, 选择合适的第二相, 构建兼具高居里温度 和高压电性能的复合压电陶瓷材料; 第二，通过实 验观测与理论计算相结合的方法研究相变机理及自

表 3 不同方法制备的 PLS 压电陶瓷的主要性能

Table 3 Characteristics of PLS piezoceramics fabricated via different methods

\begin{tabular}{|c|c|c|c|c|c|c|}
\hline Materials & Process & $T_{\mathrm{s}} /{ }^{\circ} \mathrm{C}$ & Density/\% & $T_{\mathrm{c}} /{ }^{\circ} \mathrm{C}$ & $d_{33} /(\mathrm{pC} / \mathrm{N})$ & Ref. \\
\hline $\mathrm{Sr}_{2}\left(\mathrm{Nb}_{1-x} \mathrm{Ta}_{x}\right)_{2} \mathrm{O}_{7}$ & $\mathrm{HF}$ & 1400 & 95 & 823 & 1.6 & {$[38]$} \\
\hline $\mathrm{Sr}_{2-x} \mathrm{Ba}_{x} \mathrm{Nb}_{2} \mathrm{O}_{7}$ & SPS & 1200 & 95 & 1175 & 3.6 & {$[45]$} \\
\hline $\mathrm{La}_{2-x} \mathrm{Ce}_{x} \mathrm{Ti}_{2} \mathrm{O}_{7}$ & SPS & 1400 & 95 & 1440 & 3.9 & {$[46]$} \\
\hline$\left(\mathrm{Sr}_{1-x} \mathrm{Ce}_{x}\right)_{2} \mathrm{Nb}_{2} \mathrm{O}_{7}$ & SPS & 1350 & 98 & 1327 & 1.5 & [47] \\
\hline $\mathrm{Sr}_{2}\left(\mathrm{Nb}_{1-x} \mathrm{~W}_{x}\right)_{2} \mathrm{O}_{7}$ & SPS & 1425 & 98 & 1308 & - & {$[47]$} \\
\hline$\left(\mathrm{Sm}_{x} \mathrm{La}_{1-x}\right) \mathrm{Ti}_{2} \mathrm{O}_{7}$ & SPS & 1400 & - & 1430 & 2.8 & {$[48]$} \\
\hline$\left(\mathrm{Bi}_{x} \mathrm{La}_{1-x}\right) \mathrm{Ti}_{2} \mathrm{O}_{7}$ & SPS & 1350 & 95 & 1395 & 3.6 & [49] \\
\hline $\mathrm{Sr}_{2} \mathrm{Nb}_{2} \mathrm{O}_{7}-x \mathrm{wt} \% \mathrm{La}_{2} \mathrm{O}_{3}$ & SSR & 1350 & - & - & - & {$[43]$} \\
\hline $\mathrm{Ca}_{2-x} \mathrm{Ba}_{x} \mathrm{Nb}_{2} \mathrm{O}_{7}$ & SSR & 1350 & 95 & 1280 & - & [44] \\
\hline $\mathrm{Sr}_{2}\left(\mathrm{Nb}_{1-x} \mathrm{~V}_{x}\right)_{2} \mathrm{O}_{7}$ & SSR & 1200 & 96 & - & - & {$[40]$} \\
\hline $\mathrm{Sr}_{2} \mathrm{Nb}_{2} \mathrm{O}_{7}-x \mathrm{wt} \% \mathrm{ZnO}$ & SSR & 1400 & 97 & - & - & [41] \\
\hline $\mathrm{Sr}_{2} \mathrm{Nb}_{2} \mathrm{O}_{7}-x \mathrm{wt} \% \mathrm{CuO}$ & SSR & 1180 & 98 & 1342 & 1.1 & {$[42]$} \\
\hline$(1-\chi) \mathrm{Sr}_{2} \mathrm{Nb}_{2} \mathrm{O}_{7}-x \mathrm{Na}_{0.5} \mathrm{Bi}_{0.5} \mathrm{TiO}_{3}$ & SSR & 1420 & 96.8 & 1330 & 1.0 & [51] \\
\hline
\end{tabular}

HF: Hot Forging; SPS: Spark Plasma Sintering; SSR: Solid State Reaction; $T_{\mathrm{s}}$ : Sintering Temperature 
发极化来源; 第三，探究 A 位原子对氧八面体和晶 体结构的影响, 从而找出合适的元素进行掺杂改性, 提高 PLS 压电陶瓷的压电性能。

\section{参考文献:}

[1] 董显林. 功能陶瓷研究进展与发展趋势. 中国科学院院刊, 2003, 6: $407-412$.

[2] LI Y X. Some hot topics in electroceramics research. Journal of Inorganic Materials, 2014, 29(1): 1-5.

[3] JIANG X P, KIM K, ZHANG S, et al. High-temperature piezoelectric sensing. Sensors, 2014, 14(1): 144-169.

[4] ANTON S R, SODANO H A. A review of power harvesting using piezoelectric materials (2003-2006). Smart Mater. Struct., 2007, 16(3): R1-R21.

[5] LEE H J, ZHANG S J, COHEN Y, et al. High temperature, high power piezoelectric composite transducers. Sensors, 2014, 14(8): 14526-14552.

[6] ZHANG S J, JIANG X N, LAPSLEY M, et al. Piezoelectric accelerometers for ultrahigh temperature applications. Appl. Phys. Lett., 2010, 96(1): 013506-1-3.

[7] ZHANG S J, YU F P. Piezoelectric materials for high temperature sensors. J. Am. Ceram. Soc., 2010, 94(10): 3153-3170.

[8] NIE R, CHEN Q, LIU H, et al. $\mathrm{MnO}_{2}$-doped $\left(\mathrm{Ca}_{0.4} \mathrm{Sr}_{0.6}\right) \mathrm{Bi}_{4} \mathrm{Ti}_{4} \mathrm{O}_{15}$ high-temperature piezoelectric ceramics with improved thermal stability. J. Mater. Sci., 2016, 51(11): 5104-5112.

[9] 李玉臣, 包绍明, 周志勇, 等. 一种高温下稳定使用的铋层状 结构压电陶瓷材料及其制备方法. 中国, C04B35/462, ZL200610147892.3. 2007.07.11.

[10] ZHOU Z Y, LI Y C, HUI S P, et al. Effect of tungsten doping in bismuth-layered $\mathrm{Na}_{0.5} \mathrm{Bi}_{2.5} \mathrm{Nb}_{2} \mathrm{O}_{9}$ high temperature piezoceramics. Appl. Phys. Lett., 2014, 104(1): 012904-1-4.

[11] 周志勇, 李玉臣, 董显林, 等. 提高铋层状结构压电陶瓷材料的 压电性能以及其温度稳定性的方法. 中国, C04B41/80, ZL201510107721.7. 2015.06.24.

[12] NANAMATSU S, KIMURA M, DOI K, et al. Crystallographic and dielectric properties of ferroelectric $\mathrm{A}_{2} \mathrm{~B}_{2} \mathrm{O}_{7}(\mathrm{~A}=\mathrm{Sr}, \mathrm{B}=\mathrm{Ta}, \mathrm{Nb})$ crystals and their solid solutions. J. Phys. Soc. Jpn., 1975, 38(3): 817-824.

[13] GAO Z P, YAN H X, NING H P, et al. Ferroelectricity of $\mathrm{Pr}_{2} \mathrm{Ti}_{2} \mathrm{O}_{7}$ ceramics with super high Curie point. Adv. Appl. Ceram., 2013, 112(3): 69-74.

[14] YAN H X, NING H P, KAN Y, et al. Piezoelectric ceramics with super-high Curie points. J. Am. Ceram. Soc., 2009, 92(10): 22702275.

[15] BRAHMAROUTU B, MESSING G L, TROLIER S. Densification and anisotropic grain growth in $\mathrm{Sr}_{2} \mathrm{Nb}_{2} \mathrm{O}_{7}$. J. Mater. Sci., 2000, 35(22): 5673-5680.

[16] COOK W R, JAFFE H. Ferroelectricity in oxides of fluorite structure. Phys. Rev., 1952, 88(6): 1426.

[17] JONA F, SHIRANE G, PEPINSKY R. Dielectric, X-Ray and optical study of ferroelectric $\mathrm{Cd}_{2} \mathrm{Nb}_{2} \mathrm{O}_{7}$ and related compounds. Phys. Rev., 1955, 98(4): 903-909.

[18] ROWLAND J F, BRIGHT N F H, JONGEJAN A. The Crystallography of Compounds in the Calcium Oxide-niobium Pentoxide System. Adv. X-Ray Analysis: Volume 2 Proceedings of the Seventh Annual Conference on Applications of X-Ray Analysis, 1958, 2: $97-106$.

[19] BALLMAN A A. Growth of piezoelectric and ferroelectric materials by the czochraiski technique. J. Am. Ceram. Soc., 1965, 48(2): $112-113$.
[20] BRANDONAB J K, MEGAWA H D. On the crystal structure and properties of $\mathrm{Ca}_{2} \mathrm{Nb}_{2} \mathrm{O}_{7}$, "calcium pyroniobate". Phil. Mag. A, 1970 21(169): 189-194.

[21] NANAMATSU S, KIMURA M, DOI K, et al. A new ferroelectric: $\mathrm{La}_{2} \mathrm{Ti}_{2} \mathrm{O}_{7}$. Ferroelectrics, 1974, 8(1): 511-513.

[22] KIMURA M, NANAMATSU S, KAWAMURA T, et al. Ferroelectric, electrooptic and piezoelectric properties of $\mathrm{Nd}_{2} \mathrm{Ti}_{2} \mathrm{O}_{7}$ single crystal. Jpn. J. Appl. Phys., 1974, 13(9): 1473-1474.

[23] ISHIZAWA N, MARUMO F. The crystal structure of $\mathrm{Sr}_{2} \mathrm{Nb}_{2} \mathrm{O}_{7}$, a compound with perovskite-type slabs. Acta Cryst., 1975, 31(7): 1912-1915.

[24] SYCH A M, TITOV Y A, NEDILKO C A. Synthesis and study of ferroelectric compounds with layered structures. J. Inorganic Chem., 1980, 25(8): 2056-2061.

[25] SYCH A M, TITOV Y A, NEDILKO C A, et al. Study of the conditions for the isovalent substitution of rare-earth atoms in $\mathrm{Ln}_{2} \mathrm{Ti}_{2} \mathrm{O}_{7}$ with layered structure. J. Inorganic Chem., 1987, 32(11): 2625-2628.

[26] GAO Z P, LIU L, YAN H X, et al. Cerium titanate $\left(\mathrm{Ce}_{2} \mathrm{Ti}_{2} \mathrm{O}_{7}\right)$ : a ferroelectric ceramic with perovskite-like layered structure (PLS). J. Am. Ceram. Soc., 2015, 98(12): 3930-3934.

[27] NANAMATSU S, KIMURA M, DOI K, et al. Ferroelectric properties of $\mathrm{Sr}_{2} \mathrm{Nb}_{2} \mathrm{O}_{7}$ single crystal. J. Phys. Soc. Jpn., 1971, 30(1): 300-301.

[28] ISHIZAWA N, MARUMO F. Compound with perovskite-type slabs. II. the crystal structure of $\mathrm{Sr}_{2} \mathrm{Ta}_{2} \mathrm{O}_{7}$. Acta. Cryst., 1976, 32(9): 2564-2566.

[29] NANAMATSU S, KIMURA M. Ferroelectric properties of $\mathrm{Ca}_{2} \mathrm{Nb}_{2} \mathrm{O}_{7}$ single crystal. J. Phys. Soc. Jpn., 1971, 36(5): 597-602.

[30] PATWE S J, KATARI V, SALKE N P, et al. Structural and electrical properties of layered perovskite type $\operatorname{Pr}_{2} \mathrm{Ti}_{2} \mathrm{O}_{7}$ : experimental and theoretical investigations. J. Mater. Chem. C, 2015, 3(17): 4570-4584.

[31] FILIPPETTI A, HILL N A. Coexistence of magnetism and ferroelectricity in perovskites. Phys. Rev. B, 2002, 65(19): 195120 $1-11$.

[32] BHATTACHARJEE S, BOUSQUET E, GHOSEZ P. Engineering multiferroism in $\mathrm{CaMnO}_{3}$. Phys. Rev. Lett., 2009, 102(11): $117602-$ $1-4$.

[33] CATALAN G, SCOTT J F. Physics and applications of bismuth ferrite. Adv. Mater., 2009, 21(24): 2463-2485.

[34] JORGE L P, JORGE I. Ab initio study of proper topological ferroelectricity in layered perovskite $\mathrm{La}_{2} \mathrm{Ti}_{2} \mathrm{O}_{7}$. Phys. Rev. B, 2011, 84(7): 075121-1-13.

[35] BRUYER E, SAYEDE A. Density functional calculations of the structural, electronic, and ferroelectric properties of high-k titanate $\mathrm{Re}_{2} \mathrm{Ti}_{2} \mathrm{O}_{7}(\mathrm{Re}=\mathrm{La}$ and Nd). J. Appl. Phys., 2010, 108(5): 053705$1-9$.

[36] BRAHMAROUTU B, MESSING G L, MCKINSTRY S T. Molten salt synthesis of anisotropic $\mathrm{Sr}_{2} \mathrm{Nb}_{2} \mathrm{O}_{7}$ particles. J. Am. Ceram. Soc., 1999, 82(6): 1565-1568.

[37] PRASADARAO A V, SELVARAJ U, KOMARNENI S. Sol-Gel synthesis of strontium pyroniobate and calcium pyroniobate. $\mathrm{J}$. Am. Ceram. Soc., 1992, 75(10): 2697-2701.

[38] FUIERER P A, SHROUT T R, NEWNHAM R E. Physical, electrical, and piezoelectric properties of hot-forged $\mathrm{Sr}_{2}(\mathrm{NbTa})_{2} \mathrm{O}_{7}$ ceramics. Mat. Res. Soc. Symp. Proc., 1992, 276: 51-57.

[39] GAO Z P, SUZUKI T S, GRASSO S, et al. Highly anisotropic single crystal-like $\mathrm{La}_{2} \mathrm{Ti}_{2} \mathrm{O}_{7}$ ceramic produced by combined magnetic field alignment and templated grain growth. J. Eur. Ceram. Soc., 2015, 35(6): 1771-1776.

[40] SERAJI S, WU Y, LIMMER S, et al. Processing and properties of vanadium doped strontium niobate. Mat. Sci. Eng., 2002, 88(1): 
73-78.

[41] IQBAL Y, MANAN A, SAFEEN M K, et al. ZnO as sintering additive in $\mathrm{Sr}_{2} \mathrm{Nb}_{2} \mathrm{O}_{7}$. J. Phys.: Conference Series, 2010, 241: 012029-1-4.

[42] CHEN T, LIANG R H, JIANG K, et al. Low-temperature sintering and electrical properties of $\mathrm{Sr}_{2} \mathrm{Nb}_{2} \mathrm{O}_{7}$ piezoceramics by $\mathrm{CuO}$ addition. J. Am. Ceram. Soc., 2017, 100(6): 2397-2401.

[43] FU C, LIU H, CHEN G, et al. Microstructure and electric properties of strontium lanthanum niobate ceramics. Ferroelectrics, 2012, 432(1): 8-13.

[44] LI C C, XIANG H C, QIN Y D, et al. Effects of barium substitution on the sintering behavior, dielectric properties of $\mathrm{Ca}_{2} \mathrm{Nb}_{2} \mathrm{O}_{7}$ ferroelectric ceramics. J. Adv. Diele., 2017, 7(2): 1750013-1-5.

[45] GAO Z P, NING H P, CHEN C, et al. The effect of barium substitution on the ferroelectric properties of $\mathrm{Sr}_{2} \mathrm{Nb}_{2} \mathrm{O}_{7}$ ceramics. J. Am. Ceram. Soc., 2013, 96(4): 1163-1170.

[46] GAO Z P, YAN H X, NING H P, et al. Piezoelectric and dielectric properties of Ce substituted $\mathrm{La}_{2} \mathrm{Ti}_{2} \mathrm{O}_{7}$ ceramics. J. Eur. Ceram. Soc., 2013, 33(5): 1001-1008.
[47] NING H P, YAN H X, GAO Z P, et al. Effect of donor dopants cerium and tungsten on the dielectric and electrical properties of high Curie point ferroelectric strontium niobate. Ceram. Int., 2013, 39(7): 7669-7675.

[48] CHEN C, GAO Z P, YAN H X, et al. Crystallographic structure and ferroelectricity of $\left(\mathrm{A}_{x} \mathrm{La}_{1-x}\right)_{2} \mathrm{Ti}_{2} \mathrm{O}_{7}(\mathrm{~A}=\mathrm{Sm}$ and $\mathrm{Eu})$ solid solutions with high $T_{c}$. J. Am. Ceram. Soc., 2016, 99(2): 523-530.

[49] LI C C, XIANG H C, CHEN J W, et al. Phase transition, dielectric relaxation and piezoelectric properties of bismuth doped $\mathrm{La}_{2} \mathrm{Ti}_{2} \mathrm{O}_{7}$ ceramics. Ceram. Int., 2016, 42(9): 11453-11458.

[50] TITOV V V, AKHNAZAROVA V V, REZNITCHENKO L A, et al. Evolution of fractal grain structures in $\mathrm{NaNbO}_{3}-\mathrm{Ca}_{2} \mathrm{Nb}_{2} \mathrm{O}_{7}$ and $\mathrm{NaNbO}_{3}-\mathrm{Sr}_{2} \mathrm{Nb}_{2} \mathrm{O}_{7}$ systems. Ferroelectrics, 2004, 298(1): 335-339.

[51] CHEN T, LIANG R H, LI Y C, et al. Structure and electrical properties of perovskite layer $(1-x) \mathrm{Sr}_{2} \mathrm{Nb}_{2} \mathrm{O}_{7}-x\left(\mathrm{Na}_{0.5} \mathrm{Bi}_{0.5}\right) \mathrm{TiO}_{3}$ hightemperature piezoceramics. J. Am. Ceram. Soc., 2017, 100(4): 10651072.

[52] GAO Z P, LU C J, WANG Y H, et al. Super stable ferroelectrics with high Curie point. Sci. Rep., 2016, 6: 24139-1-6. 